\title{
Capítulo II Comprensión de la dimensión y las armas de la ciberguerra
}

ntes de iniciar este capítulo de manera formal en cuanto al tema
bien el caquítulo pratará, es relevante hacer una aclaración previa. Si
de la guerra, estableciendo algunos aspectos tanto definitorios como
característicos de esta práctica, cabe señalar que este no va a presen-
tar la misma estructura en relación con lo mencionado. Es un capítu-
lo que se constituirá más desde el enfoque que tiene el primero en su
segunda parte, es decir, donde se intenta construir la idea de que la
guerra frente a un concepto dimensional ha sido una cuestión tanto
evolutiva como paralela. Y se quiere ver aśí, en últimas, que todas las
dimensiones de la guerra y todos sus poderes siguen desarrollándose
continuamente hoy, así que, si bien en algún momento de la historia
primaron algunos escenarios, actualmente la cuestión sigue siendo
transversal o se presenta como transversal.
No obstante, asumiendo esta perspectiva, desde un concepto más
consecutivo o evolutivo, el presente capítulo tiene su fundamento, en
primera instancia, en recalcar la idea de que la consecución o cons-
trucción del ciberespacio, y su determinación como un espacio viable
para generar nuevas actividades de violencia o fuerza enmarcada den-
tro del concepto de la guerra, fue el momento en el cual precisamente
esta práctica encontró una nueva dimensión para extender sus límites. 
Por esto, se asumirán algunas definiciones ya desde una perspectiva de la seguridad y de lo que significa el ciberespacio para la seguridad nacional de los Estados. Por supuesto, se seguirá manejando una lógica teórica y conceptual y el foco estará más en una explicación de fenómeno que de casos.

En una segunda instancia, se proporcionará una explicación desde la perspectiva del espacio de lo que significa el concepto de centro de gravedad, que fue explicado en el capítulo anterior y con el cual se llegó a la conclusión de que, a través del ejercicio de la guerra, más allá de querer aniquilar o destruir a un pueblo, aunque en ciertos casos de la historia sí haya sido ese el motivo, lo que esperan los estrategas militares es poder atacar a aquello que es de vitalidad para su enemigo y su continuidad en el campo de batalla. Al llegar al capítulo enfocado en los casos y las formas de ciberguerra, el lector observará que en muchos de ellos el ejercicio de lo que es denominado ciberguerra ya no se encuentra relacionado, como el término mismo lo indica, con un espacio de guerra o con una condición de guerra, sino que muchas veces lo que está de por medio es un conflicto de intereses que son vitales para los Estados nación.

En última instancia, se analizará qué es lo que se puede entender como armas dentro del ciberespacio, pues, a partir de lo planteado en el capítulo 1, poder hablar de guerra desde la perspectiva que interesa a este libro significa la sumatoria de varios elementos. Primero, y como es justo aclarar en este momento, implica la existencia de unas dimensiones que desde la característica clásica fueron dispuestas de manera natural por el planeta Tierra. Por ende, el primer elemento que se debe tener en cuenta es un espacio en el cual se puede desarrollar la práctica de la guerra —en este caso ya se hará hincapié en el ciberespacio como un nuevo campo de batalla-.

Segundo, y en un sentido lógico, al hablar de una práctica bélica que implica el uso de la fuerza y la violencia por parte de los Estados y sus ejércitos, y como se analizará al finalizar el libro, de otro tipo de actores no estatales que ya han sido reconocidos como ejecutores de guerra, se deberá analizar otro elemento: cuáles son las armas que se llevan al ciberespacio y cómo se configuran como armas al permitir ese objetivo militar de atacar aquello que es importante para un Estado enemigo o 
un Estado que puede estar atentando contra los intereses o la seguridad de una nación.

Si aún existen cuestionamientos sobre por qué no se ha definido la ciberguerra, el presente libro, para seguir una línea lógica de lo expuesto en el capítulo anterior, considera preponderante entender, primero, el escenario en el cual se lleva a cabo toda la acción ciberespacial y cuáles son las herramientas que se pueden usar, es decir, dónde y con qué se lleva a cabo la guerra cibernética, para luego concebir el qué, esto es, como fenómeno y cuáles son sus características, sin necesidad de desviarse en conceptos clave para comprenderla. Por tanto, la intención es entender el contexto para luego poder sumergirse estrictamente en este y las clasificaciones que de él se derivan.

De igual forma, y con el propósito de lograr ubicar al lector, una definición general de ciberguerra es propuesta por Arquilla y David Ronfeldt (1997), en la cual se afirma que es

conducir y prepararse para llevar a cabo operaciones militares de acuerdo con los principios relacionados con la información. Significa interrumpir, si no se logra destruir, los sistemas de información y comunicación, que ampliamente definidos incluyen la cultura militar, es decir, aquella información en la que un adversario confía para operar de forma correcta: quién es, dónde está, qué puede hacer en diferentes ocasiones, por qué iniciar un conflicto, qué contraatacar primero, etc. Significa tratar de saber todo acerca de un adversario mientras se evita que sepa mucho sobre el atacante. Significa poner el equilibrio de información y conocimiento a favor propio, en especial si el equilibrio de fuerzas no lo está. Significa usar el conocimiento para que haya que gastar menos capital y trabajo ${ }^{10}$. (p. 30)

En los siguientes capítulos, se hará un análisis mucho más detallado y completo de lo que representa la ciberguerra, y los cambios que esta ha generado.

10 Traducción del autor. 


\section{El ciberespacio como dominio}

Con la globalización de las tecnologías informáticas y la consecuente configuración del ciberespacio, se estructuró un escenario para trasladar la lógica de la guerra. Como ya se formuló hace varios años, se podría entender el ciberespacio como la representación de la quinta dimensión de la guerra, o bien, como lo hizo The Economist (2010), el quinto dominio de batalla. Asumiendo este concepto inicial, se valida el trabajo contextual del capítulo anterior, pues los analistas no solo reconocen otros espacios previos para la guerra, sino que, además, asumen la existencia del ciberespacio como uno más, lo cual es fundamental para evidenciar si existe o no una forma de guerra en esta dimensión.

El Departamento de la Defensa de los Estados Unidos (United States Department of Defense [DoD]) fue uno de los primeros organismos gubernamentales en conceptualizar este nuevo territorio de la guerra. Sus concepciones se encuentran desde aceptarlo como un entorno en el que la información digitalizada se comunica a través de redes informáticas, hasta formas más complejas como definiciones que lo presentan como un dominio caracterizado por el uso de la electrónica y del espectro electromagnético. Para 2008, una "nueva" definición caracterizó el ciberespacio como el dominio global dentro del entorno de la información que consiste en la red interdependiente de infraestructuras de tecnologías de la información y de la comunicación (TIC), incluida internet, redes de telecomunicaciones, sistemas informáticos, y los procesadores y controladores incrustados (Singer y Friedman, 2014).

Para Ortega, el ciberespacio es el espacio artificial constituido por el conjunto de redes de ordenadores y de telecomunicaciones interconectados directa o indirectamente a nivel mundial, creado por el hombre para ser usado a su servicio. De manera más concreta, es

un dominio global dentro del entorno de la información cuyo carácter distintivo y único está enmarcado por el uso de la electrónica y el espectro electromagnético para crear, almacenar, modificar, intercambiar y explotar información a través de redes interdependientes 
e interconectadas utilizando tecnologías de la información y de la comunicación. (2012, p. 27)

Asimismo, el ciberespacio se ha configurado como el entramado de tecnologías informáticas que se constituye en el pilar sobre el que se erige y sustenta un medioambiente virtual de información y de continua interacción humana y artificial que transgrede el espacio geográfico y las barreras temporales de comunicación y forma parte de todas las actividades diarias del mundo (Trias y Bell, 2010). Las ideas de Trias y Bell suman elementos de análisis importantes, demuestran de manera implícita que, a diferencia de las otras dimensiones en las que se trasladó la guerra, esta ya posee la capacidad de no ser geográfica, no estar limitada por barreras físicas o políticas, y el tiempo de las actividades que allí se desarrollan en relación con el tiempo distan de cuando se hacen en el mundo real.

Si bien Singer y Friedman (2014) concuerdan con una definición técnica en la que se comprende el ciberespacio como un ámbito donde las redes que están compuestas por diversas computadoras, al igual que los usuarios que explotan sus capacidades, y a través de las cuales se almacena, comparte y comunica en línea la información, desde lo político, social y estratégico, es preferible acoger definiciones como la emitida por la Casa Blanca de los Estados Unidos en el año 2003, en la que el ciberespacio representa más el sistema de control del país, ya que está "compuesto por cantidades inmensurables de computadoras, servidores, routers, conmutadores y cables de fibra óptica, que, al encontrarse interconectados, permiten el correcto funcionamiento de las infraestructuras críticas"11 (p. 1). Esto lleva a pensar que el ciberespacio, así como denota capacidades, simultáneamente significa riesgo (Stel, 2014).

Además de las posibles definiciones que se puedan exponer del ciberespacio, es posible afirmar que es una dimensión tanto para la guerra como para procesos no bélicos, que integra el conjunto de dimensiones precedentes - la terrestre, marítima, aérea y espacial- debido al número infinito de conexiones que en la actualidad se generan

11 Traducción del autor. 
desde estos escenarios hacia el ciberespacio y como producto de que muchas de las herramientas, maquinarias, vehículos, etc., dependen de información para funcionar. Anteriormente, se abordó la capacidad de integración de las tecnologías, así que, si bien los carros, barcos y aviones se fusionaron con la artillería, muchas de las cosas que se usan en la vida cotidiana como en la guerra se fusionaron con la informática y la automatización.

Como consecuencia, el ciberespacio, en materia de los procesos humanos que permite realizar, evoluciona a una velocidad abismal debido al desarrollo tecnológico exponencial que constantemente lo alimenta, y el número de recursos que lo conforman, al igual que el de usuarios que lo explotan, aumenta sin desaceleración (López de Turizo y Sánchez, 2012).

Lo anterior, en paralelo a la referencia de Stel (2014) frente al riesgo, conduce a una realidad que será sustentada en los próximos capítulos. El ciberespacio logra trascender los límites geopolíticos y es bastante acertado en el momento de integrar las operaciones de la infraestructura crítica, de gobernabilidad, de comercio y, desde luego, de seguridad nacional (Gorman, 2005). Las cosas que están conectadas al ciberespacio, pero que hacen parte de las otras dimensiones de la guerra, también serán vulnerables frente a la ciberguerra.

No fue fortuito, entonces, que algunos ejércitos, los de los países militarmente desarrollados, al finalizar la década de 1990 comenzaran a discutir, teorizar y adoctrinar en lo que para el momento se llamó la information war e information warfare (IW). Asimismo, formaron una red de computadoras en un sistema integrado de comunicación (Dunn, 2001). En torno a estas prácticas, conformaron grupos especializados con capacidades informáticas para desarrollar una defensa y una ofensiva de carácter estratégico. Esto desencadena el análisis de algunos aspectos como la revolución de los asuntos militares en torno a esta evolución de la guerra gracias a la introducción de dispositivos que mejoraron la transmisión de información, así como la integración de equipo y armas preexistentes con dispositivos informáticos.

Aun así, en el contexto de la ciberguerra que invita este libro, la información como recurso y elemento se tratará como determinante en la guerra contemporánea. Pero se ha desarrollado otro escenario a 
partir de las tecnologías informáticas y el ciberespacio, que en realidad da vida a lo que se denomina ciberguerra, y es donde las tecnologías informáticas se fundamentan a partir de los principios de la cibernética (Bieber, 2000).

Gracias a la ciberguerra, tanto la guerra interestatal como la guerra irregular se han traslapado al ciberespacio. El ciberespacio y las tecnologías que dan acceso a él han posibilitado que los ataques que se llevan a cabo en la virtualidad se pueden traducir en destrucción, caos suntuario, víctimas mortales y sociedades paralizadas, como si los Estados desplegaran sus ejércitos terrestres, fuerzas aéreas y armadas marítimas sobre su enemigo para ejercer fuerza y poder. Podría significar, de la misma manera, equilibrio al comprender que los terroristas, en vez de detonar sus artefactos explosivos o secuestrar aviones, emplearan el ciberespacio contra una nación.

La evolución del ciberespacio ha configurado nuevos escenarios para el desarrollo de los conflictos en el siglo xxi (Simonetti, 2008). Los países han explotado las posibilidades que ofrece el ciberespacio para realizar acciones como el ciberespionaje, que hace parte de un primer nivel en el cual se asume que la información es el fin, donde el objetivo de las operaciones es buscar información vital desde la perspectiva del perpetrador. Por dar solo un ejemplo, hay evidencias contundentes que demuestran que China ha realizado ciberespionaje a potencias occidentales desde 2002 dentro de lo que se denominó la operación Titan Rain (The U.S.-China Economic and Security Review Commission, 2009).

Las tecnologías informáticas se han vuelto estratégicas para acciones como el ciberespionaje, gracias a que presentan capacidades que inhabilitan los métodos y las tecnologías que las autoridades de muchos Estados han dispuesto para evitar este tipo de incidentes. Y, por ende, no hay forma de proponer herramientas eficientes de trazabilidad que permitan al actor agredido presentar pruebas contundentes frente a la violación de soberanía acaecida.

Es decir, el ciberespacio se ha descrito como una red interdependiente de sistemas de tecnologías informáticas, entre las que se denotan las computadoras o los procesadores, internet y los sistemas de control de la infraestructura crítica del Estado (U.K. Office of Cyber Security, 2009). 
En general, una clara representación de lo anterior es que los gobiernos se preocupan constantemente por invertir en la construcción de e-government para generar una cercanía con la ciudadanía y alcanzar estándares óptimos de gestión, que los flujos de valores y capital circulen por el globo gracias a la interconexión de las bolsas y los mercados nacionales, que los sistemas informáticos de control de infraestructuras críticas del Estado y privadas se puedan controlar y monitorear a distancia, que los controladores de tráfico terrestre y aéreo funcionen en internet, que el sector privado y las personas apoyen sus actividades rutinarias en las capacidades de las TIC, entre otros casos que se pueden destacar (Gorman, 2005).

\section{Extrapolación de los centros de gravedad de la guerra al ciberespacio}

Sampaio (2001) propone que, tras su aparición, ha existido una dependencia entre el ser humano social y el ciberespacio, este expresado en sus recursos tangibles e intangibles, con la intención de facilitar los procesos cotidianos. Pero esta relación ha sido nociva por las potenciales vulnerabilidades que ha creado en la seguridad de los Estados y todos los actores que de él dependen, lo que abre fisuras que pueden ser penetradas por un enemigo para atacar las estructuras que el Estado haya asignado al control del ciberespacio, afectándolo física, moral y psicológicamente.

Esta capacidad tecnológica puede significar el dominio en un nuevo espectro, alejado de las armas tradicionales que poseen los Estados y las fuerzas militares y su superioridad convencional, atrae nuevas amenazas, pero no para las tropas que están en el frente de batalla, sino a cualquier sistema, de carácter gubernamental, militar, económico o social, que ahora haga parte del ciberespacio, así esté a kilómetros del campo de acción (Smith, 2004).

Si se tiene en cuenta a John Warden y su teoría sobre la existencia de cinco anillos, el panorama es más claro. Warden, en esencia, tiene la idea de que el Estado se encuentra compuesto por cinco anillos donde cada uno representa una parte vital de este, los cuales 
se traducen en los mecanismos de combate, la población, la infraestructura crítica, los elementos orgánicos y sintéticos esenciales y los líderes gubernamentales (Fadok, 1995).

De esta manera, cuando se aterriza sobre el escenario descrito, donde los nódulos de subsistencia de una nación se encuentran integrados al ciberespacio, y sabiendo ya que se pueden desarrollar ciberataques con las tecnologías informáticas, se aclara cómo, desde la teoría de Warden, la estructura del Estado se encuentra en gran riesgo, ya no por las capacidades del poder aéreo como fue construido el postulado originalmente, sino por las capacidades que ofrecen las computadoras y el ciberespacio cuando los anillos se encuentran vinculados a estos.

Warden llevó el principio de centro de gravedad a una nueva instancia al observar al enemigo como un sistema organizado en anillos concéntricos. En conexión al concepto clásico, cada anillo de Warden representa un centro de gravedad diferente del enemigo (Jackson, 2000, p. 3). En un orden de importancia comprendido a partir del centro al exterior de los anillos, se identifica lo siguiente: un anillo de liderazgo que controla el sistema o Estado, es decir, los líderes del Estado; uno de sistemas esenciales que proporciona o representa la producción clave que es crítica para la supervivencia del Estado, esto es, el petróleo, la electricidad, la comida o el dinero; un tercer anillo de infraestructura crítica que une todo el sistema, como el transporte; luego el anillo de la población civil del Estado; y, por último, el anillo de las Fuerzas Armadas de los Estados. Warden cree que el objeto de la guerra es inducir al enemigo a hacer su voluntad; y se puede lograr de manera más eficaz y eficiente mediante ataques rápidos y simultáneos contra el anillo interior del enemigo (liderazgo). Solo si uno es incapaz de atacar el liderazgo de un enemigo, Warden recomienda atacar, en orden ascendente de importancia, los demás anillos (Jackson, 2000, p. 4) (véase figura 1).

Para que sea aún más fácil entender el alcance de estos potenciales riesgos, los Estados Unidos son un fiel ejemplo: casi la totalidad de las comunicaciones en el sector militar y de defensa son transmitidas por redes comerciales, que pueden ser interceptadas expeditamente. Además, en muchos casos, los insumos tecnológicos son comprados en Estados con intereses contrarios a los del país norteamericano y, por 
supuesto, en múltiples casos, los archivos sobre temas delicados, secretos o los mismos avances tecnológicos ya se encuentran digitalizados y podrían ser manipulados o robados por actores que logren infiltrarse en los sistemas de seguridad cibernéticos (Bishop y Goldman, 2010).

Figura 1. Los cinco anillos de John Warden

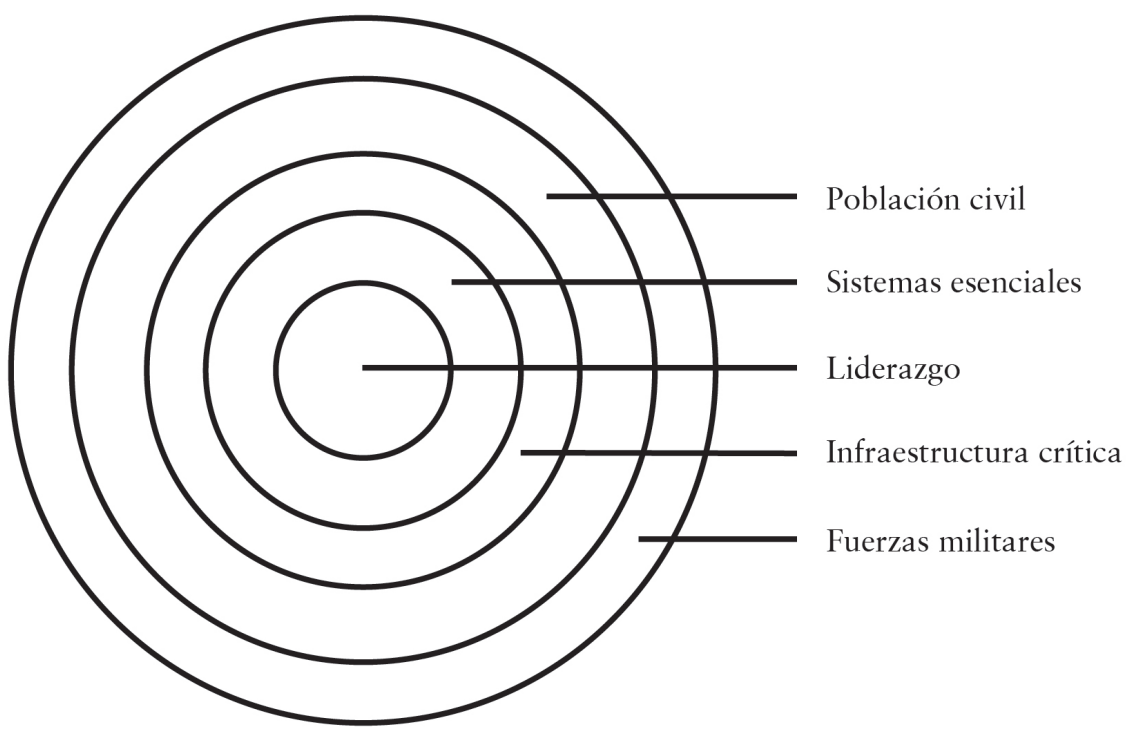

Fuente: Elaboración propia a partir de Jackson (2000).

Esta realidad no es solo de los Estados Unidos o los países con mayores recursos o niveles altos de desarrollo. Debido a los bajos costos de las tecnologías informáticas y los servicios de redes, se convirtió en una realidad para millones de usuarios en el mundo, que la usan para conseguir sus intereses de una forma más inmediata. Al ser un proceso en constante crecimiento, que parece no detenerse en el futuro próximo, se podría hablar de un paradigma distinto de cualquier conocido, que transformó la vida de los Estados y de los ciudadanos en algo nunca antes visto (Estupiñán, 2001, p. 1). Pero ¿cómo ocurrió esto? Si bien es cierto que el ciberespacio es algo virtual, cabe preguntarse cómo se puede distinguir qué es lo que lo sustenta desde la realidad. 
Como dato curioso e introductorio, Asaolu (2006) señala que, mientras que la radio tardó treinta años en llegar a más de sesenta millones de personas y la televisión tardó quince, internet, a partir de 1991, alcanzó esta meta tan solo en tres años (p. 338). Pero, asimismo, el número de usuarios de internet aumentó diez veces de 1999 a 2013. Los primeros mil millones se alcanzaron en 2005. Los dos mil millones en 2010 y los tres mil millones en 2014 (cf. Internet Live Stats).

El Pew Research Center encontró en 1995 que el $14 \%$ de los adultos estadounidenses ya estaban conectados a internet para ese momento, la mayoría usando conexiones de módem de acceso telefónico. En 1996, el 77 \% de los usuarios en línea envió o recibió correos electrónicos al menos una vez cada par de semanas, frente al $65 \%$ de 1995. En 1998, el primer índice de Google hace referencia a veintiséis millones de páginas web; para 2000, Google rompió el récord de un billón de páginas (Press, 2015).

En 2003, se realiza la primera Cumbre Mundial sobre la Sociedad de la Información en Ginebra; en 2004, la banda ancha supera al dialup; en 2005, se alcanzan los mil millones de usuarios de internet, el DSL es más veloz y se introducen los cables 3.0; en 2007, se hace el lanzamiento del primer iPhone; en 2008, los países en desarrollo tienen el $50 \%$ de los usuarios de internet del mundo (InternetSociety, 2014).

Entre 2007 y 2008, el ciberespacio cobró una relevancia inesperada en otra época: por primera vez un país usó internet para realizar unas votaciones, Estonia. El $36 \%$ de la población norteamericana hacía sus consultas en Wikipedia y el índice de Google alcanzó más de un trillón de direcciones únicas. En 2009, se realiza el primer lanzamiento de la red 4G; en 2010, la banda ancha móvil supera a la fija, Wikipedia alcanza los mil millones de ediciones y existen quinientos millones de abonados de banda ancha fija. Para 2011, el $50 \%$ de los usuarios de internet tienen una conexión de banda ancha móvil; en 2012, los videos componen el $50 \%$ del tráfico de internet y los países en desarrollo tienen más del $50 \%$ de abonados a la banda ancha móvil del mundo; en 2014, los teléfonos inteligentes representan el $80 \%$ de todos los teléfonos móviles (Internet Society, 2014). Para 2014, el número de usuarios de internet llegaba a los tres mil millones; actualmente alcanza casi los cuatro mil (Press, 2015). 
En 2014, casi el $75 \%$ (2,1 mil millones) de todos los usuarios de internet del mundo (2,8 mil millones) viven distribuidos en solo 20 países. El restante 2,5\% (700 millones) se distribuyen entre los otros 178 países, cada uno representando menos del 1\% del total de usuarios. China, el país con más usuarios (642 millones en 2014), representa casi el $22 \%$ del total y tiene más usuarios que los tres países siguientes (los Estados Unidos, la India y Japón). Entre los 20 países con mayor penetración, la India es el de menor con el $19 \%$, pero en el que se aumenta con mayor velocidad anualmente. En el extremo, los Estados Unidos, Alemania, Francia y Canadá tienen la mayor penetración: más del $80 \%$ de la población en estos países tiene conexión a internet (Unión Internacional de Telecomunicaciones, 2014). En lo respectivo al uso por regiones a nivel mundial, el mayor uso se da en Asia, con un 48,4\%; le sigue el continente americano, con un 21,8 \%; Europa, con un $19 \%$; África, con el 9,8\%; y Oceanía culmina las estadísticas con un $1 \%$ (cf. Internet Live Stats) (véase tabla 1).

Tabla 1. Uso de internet por regiones alrededor del mundo

\begin{tabular}{|l|r|r|r|}
\hline \multirow{2}{*}{} & \multicolumn{2}{|c|}{ Total de usuarios } & \multirow{2}{*}{ Crecimiento (\%) } \\
\cline { 2 - 3 } & 2000 & \multicolumn{1}{c|}{2017} & 7722,1 \\
\hline África & 4514400 & 353121578 & 1539,6 \\
\hline Asia & 114304000 & 1874136654 & 4220,9 \\
\hline Medio Oriente & 3284800 & 141931765 & 2035,8 \\
\hline $\begin{array}{l}\text { América Latina y el } \\
\text { Caribe }\end{array}$ & 18068919 & 385919382 & \\
\hline
\end{tabular}

Fuente: Elaboración propia a partir de Internet Live Stats.

Más de la mitad del mundo ahora usa un teléfono inteligente, casi dos tercios de la población tiene uno, más de la mitad del tráfico web del planeta proviene de estos dispositivos, más de la mitad de todas las conexiones móviles son de banda ancha y, de cada cinco personas en el globo, al menos una ha comprado en línea durante los últimos treinta días (Kemp, 2017).

Los teóricos de la materia han establecido que, si bien el ciberespacio puede catalogarse como un escenario donde el Estado puede ver amenazada su soberanía, es debido a que de manera similar 
a como se constata en los poderes militares preexistentes, al utilizar las tecnologías informáticas como armas en el ciberespacio, se está recreando el tradicional empleo de un dispositivo bélico en una dimensión de conflicto y con el fin de propiciar un efecto en el enemigo (Wilson, 2007). Algo muy cercano a lo observado con Clausewitz (1942) en el capítulo 1.

Cuando se analiza el ciberespacio desde una posición netamente guerrera, no se puede cometer el error de calificarlo de un espacio aislado y distante de la realidad física conocida de otros dominios, como los conflictos terrestres, aéreos o marítimos. Del mismo modo que un enfrentamiento convencional puede llegar a ser catastrófico, la intención del uso de tecnologías es el mismo, causar el mayor daño posible, pero con una diferencia importante: en múltiples casos, pueden existir efectos colaterales, tanto a terceros actores (civiles) como a propios, y el ciberespacio permitiría disminuir casi por completo esta consecuencia al afectar directamente los centros de gravedad del contrario (Ottis, 2010).

Sería una falla por parte de los actores estatales menospreciar los alcances de los enfrentamientos en un contexto ciberespacial. En la actualidad, las estructuras más importantes y sensibles pueden ser manejadas a kilómetros de distancia, lo que pone al alcance dañar centrales nucleares, enviar comandos errados a los medios de transporte y mensajes equivocados a los medios de comunicación, hacer colapsar las entidades financieras, entre otros múltiples efectos devastadores, y en este caso ya no únicamente al sector militar o gubernamental, sino también a la población y al Estado como un todo (Geers, 2009).

En definitiva, un número importante de países se ha percatado de la realidad del ciberespacio como un nuevo campo de batalla. Esta nueva dimensión del poder militar exige una concepción estratégica, operacional y táctica. Además, el desarrollo de una cultura que reconozca la importancia de las tecnologías informáticas en relación con la seguridad nacional. En conclusión, los Estados deben prepararse para librar la ciberguerra y ganarla (Clark y Knake, 2010).

Castells (2004) se ha convertido en un teórico indispensable para entender el mundo anclado a la tecnología, afirmando que existe una relación innegable y casi irrompible entre el comportamiento cotidiano 
de todos los actores sociales (Gobierno, sector privado, ciudadanía) y el ciberespacio. Las facilidades que ofrece internet sobre los procesos que podían tardar años, la conexión, la inmediatez, la cercanía, entre otras, han hecho que los sistemas y las redes informáticas sean demandados en cualquier rincón del planeta sin necesidad de insertarlos a la fuerza.

Habiendo generado elementos para sustentar que gran parte de los procesos humanos, no solo la guerra, se enlazaron con las tecnologías informáticas, se retomará el concepto de centros de gravedad para terminar de exponer por qué el ciberespacio se convirtió en un nicho apropiado para una práctica bélica; desde otra perspectiva, en la actualidad, se asume el hecho de que los centros de gravedad no solo podrían ser atacados mediante el armamento convencional, o improvisado de los actores irregulares, sino también mediante computadoras y personas expertas que pueden usarlos en un sentido violento.

Desde la perspectiva de Anding, uno de los principios más ampliamente reconocidos del arte operativo es el centro de gravedad. Y aunque sus raíces se encuentren a casi doscientos años en el tiempo, cuando Clausewitz recreó el concepto, este ha perdurado (2007, p. 3).

Recientemente, se redefinió el concepto de centro de gravedad como aquellas características, capacidades o localidades de las que una fuerza militar deriva su libertad de acción, fuerza física o voluntad de luchar, donde el camino más rápido a la victoria es destruyéndola o neutralizándola. El Cuerpo de Marines de los Estados Unidos describe los centros de gravedad como "fuentes importantes de fuerza". Dependiendo de la situación, pueden ser características intangibles como la determinación o la moral, o capacidades tales como fuerzas blindadas o la fuerza de aviación. Pueden ser localidades tales como un pedazo de terreno crítico que ancla un sistema defensivo entero. Pueden ser la relación entre dos o más componentes del sistema, como la cooperación entre dos armas, las relaciones en una alianza o la unión de dos fuerzas. El centro de gravedad es una fuente de fuerza masiva, física o moral, o una fuente de influencia, cuya grave degradación, dislocación, neutralización o destrucción tendría el impacto más decisivo sobre la capacidad del enemigo o su propia capacidad para lograr un objetivo militar determinado (Anding, 2007, p. 4). 
De nuevo citando a Anding, se pueden destacar algunas características de los centros de gravedad:

Los centros de gravedad tienen elementos tangibles e intangibles; cuanto mayor sea el nivel de guerra, menor será el número de centros de gravedad. En general, hay uno a nivel estratégico; los centros de gravedad operacional suelen incluir aspectos más tangibles del poder militar. Los ejemplos incluyen un batallón blindado, un grupo de portaaviones o un sistema de defensa aérea integrado; el número de centros de gravedad operacional está directamente relacionado con el número de objetivos operacionales; finalmente, los centros de gravedad no son lugares ni son capacidades. Poseen capacidades y se benefician de ciertos lugares $^{12} \cdot(2007$, p. 5$)$

Según Strange, los centros de gravedad poseen una estructura interna de varios componentes que los convierten en sistemas más complejos que protegen de las amenazas. Entre estos componentes, están las capacidades críticas, habilidades primarias que los erigen como tales, y que se relacionan con el poder físico, mental, financiero o legal para realizar una función. Segundo, los requerimientos críticos: estos son condiciones, recursos y medios esenciales para que una capacidad crítica sea plenamente operativa. Tercero, la vulnerabilidad crítica: elementos o componentes vulnerables ante la neutralización, interdicción o ataque (daño moral/físico), con lo que se logran deterioros decisivos (2005, p. 43).

Como se observó, se puede hablar de una clasificación doble para los centros de gravedad: morales y físicos. Los morales comprenden a los líderes, quienes toman las decisiones, y cómo las operaciones pueden hacer cambiar su comportamiento, o pueden ser atacados de forma directa. Y por otra parte, el apoyo público, popular (entendido como la población en general) o nacional (relativo al concepto de nación o Estado como un todo). Los centros de gravedad físicos se dividen en tres categorías. La primera categoría son las Fuerzas Armadas, la fuerza y el poder en todos los niveles de la guerra. Las categorías

12 Traducción del autor. 
segunda y tercera pertenecen al nivel estratégico: poder económico o industrial nacional, de modo que son los cimientos de la fuerza física nacional, y el poder proveniente de grandes poblaciones (Strange, 2005 , p. 45). Para la ciberguerra, conviene tener en cuenta un aspecto más señalado por Strange:

Los centros de gravedad morales en todos los niveles, y los políticos a nivel estratégico, hacen que las cosas sucedan en virtud de su voluntad, influencia y liderazgo. Estos están basados en las personas, como individuo y como población. Los centros de gravedad morales y políticos deben poseer cualidades tales como determinación, coraje (moral y físico), y el poder de persuadir, inspirar o intimidar. Los centros de gravedad físicos a nivel estratégico pueden incluir fuentes directas o centros de fuerza militar, así como las principales fuentes indirectas de esa fuerza para incluir el poder económico e industrial y el poder que provienen de las grandes poblaciones nacionales. En los niveles operativos y tácticos, son fuentes primarias y centros de fuerza militar (es decir, unidades y formaciones militares), que hacen que las cosas sucedan en virtud de su poder militar ${ }^{13}$. $(2005$, p. 47)

Eikmeier expone que los actores político-militares tienen en cuenta cuatro principios para determinar los centros de gravedad en sus enemigos. El primero es determinar la capacidad crítica del enemigo, la función absolutamente esencial que el sistema de este realiza. El sistema puede tener varias capacidades, pero no todas resultan críticas en cada situación. Segundo, identificar la fuente de poder crítico del enemigo, que es su centro de gravedad. Tercero, identificar los requisitos críticos del centro de gravedad. Y, finalmente, identificar los requisitos o componentes críticos que son vulnerables a ataques o interrupciones (2004, p. 3).

Se continuará el análisis de las razones por las que el ciberespacio se ha concebido como la quinta dimensión de la guerra. Los países son pirámides invertidas que descansan precariamente en sus entrañas

13 Traducción del autor. 
estratégicas: liderazgo, comunicaciones, producción esencial, infraestructura y población. Si un país está paralizado estratégicamente, es derrotado, y no puede sostener sus fuerzas en el campo, aunque estas estén completamente intactas (Jackson, 2000, p. 3).

Por elementos esenciales del sistema se hace referencia a aquellos atributos que a menudo son necesarios para que el Estado emprenda la guerra. Como señala Warden (1995),

los principales ejemplos de lo esencial del sistema incluyen la fuente de energía de un Estado, es decir, la electricidad, el petróleo, los alimentos y los recursos financieros. Si bien la destrucción de estos objetos puede privar al Estado enemigo de su capacidad de hacer la guerra, tal destrucción a menudo amenaza la supervivencia de la población civil del enemigo. Es este último punto el que causa las mayores preocupaciones legales y morales al orientar los elementos esenciales del sistema [...]. Cobra así relevancia el concepto de infraestructura crítica, el cual se refiere a aquellos atributos que unen el sistema y lo ayudan a funcionar como un único organismo o entidad. Las carreteras, los aeródromos y las fábricas son parte de la infraestructura crítica. (citado por Jackson, 2000, pp. 18-20)

La concepción del centro de gravedad con relación a la infraestructura crítica ha sido tema de interés para los Estados. A manera de ejemplo, el Instituto Nacional de Estándares y Tecnología (National Institute of Standards and Technology [NIST]) define las infraestructuras críticas como aquellos sistemas y activos, físicos o virtuales, tan vitales que su incapacidad o destrucción tendría un impacto debilitante en la seguridad nacional, salud pública o seguridad, o cualquier combinación de estas. Asimismo, la Comisión Europea describe las infraestructuras críticas como instalaciones, redes, servicios y activos físicos y de tecnología de la información que, de ser perturbados o destruidos, tendrían un grave impacto en la salud, la seguridad o el bienestar económico de los ciudadanos o el funcionamiento efectivo de los gobiernos (Colesniuc, 2013, p. 123).

En la misma línea, como lo proponen Alcaraz y Zeadallay (2015), una infraestructura crítica consiste en un conjunto de sistemas y activos físicos o virtuales, tan esenciales para la nación que cualquier 
interrupción de sus servicios podría tener un impacto serio en la seguridad nacional, el bienestar económico, la salud pública, o cualquier combinación de estos (p. 53).

En otro sentido, la protección de infraestructura crítica debe asumirse en términos prácticos y concretos, como la protección de la energía, de las telecomunicaciones, el suministro de agua, transporte, finanzas, salud y otras infraestructuras que permiten que una nación funcione. Estas infraestructuras críticas deben ser protegidas contra eventos accidentales y deliberados que les impidan funcionar de manera correcta, afectando así gravemente el bienestar económico y social de esa nación (Kellermann, Martinez, Contreras y Marchiori, 2015 , p. 13). En la mayoría de los países, la palabra crítica se refiere a la infraestructura que proporciona un apoyo esencial para el bienestar económico y social, la seguridad pública y el funcionamiento de las responsabilidades gubernamentales clave (Organisation for Economic Cooperation and Development [OCDE], 2008, p. 3).

La infraestructura crítica puede incluir comunicaciones, servicios de emergencia, energía, represas, sector financiero, comida, servicios públicos, industria, salud, transporte, gas, comunicaciones públicas, radio y televisión, tecnologías de la información, instalaciones comerciales, centros químicos y nucleares, y agua. Muchos Estados dependen cada vez más de infraestructuras y bienes que se encuentran parcial o totalmente fuera de su jurisdicción y sobre los cuales tienen poco o ningún control (Counter-Terrorism Committee Executive Directorate [CTED], 2017, p. 2).

En un sentido más detallado, siguiendo de nuevo a Alcaraz y Zeadallay, se presentarán los elementos del orden nacional considerados críticos:

- Energía: fuentes energéticas, almacenamiento y distribución (petróleo, gas, electricidad).

- Tecnologías de la información y las telecomunicaciones: sistemas de información y protección de la red (por ejemplo, internet), suministro de telecomunicaciones fijo, prestación de telecomunicaciones móviles, comunicación por radio y navegación, comunicación por satélite, radiodifusión. 
- Agua: suministro de agua (por ejemplo, presas) y control de calidad y cantidad de agua.

- Alimentación y agricultura: provisión de alimentos, seguridad y protección.

- Salud y salud pública: atención médica y hospitalaria, medicamentos, vacunas y productos farmacéuticos, biolaboratorios y bioagentes.

- Sistemas financieros: banca, servicios de pago y asignación financiera del Gobierno.

- Administración civil: instalaciones y funciones gubernamentales, Fuerzas Armadas, servicios de administración civil, servicios de emergencia, servicios postales y de mensajería.

- Orden público, ordenamiento jurídico y seguridad: mantenimiento del orden público y jurídico, seguridad y protección, administración de justicia y detención.

- Sistemas de transporte: transporte por carretera, transporte ferroviario, tránsito aéreo, vigilancia de fronteras, transporte fluvial.

- Industria química: producción y almacenamiento de sustancias peligrosas, tuberías de mercancías peligrosas.

- Monumentos e íconos nacionales: monumentos, estructuras físicas, objetos o lugares geográficos reconocidos como representativos de la cultura nacional o que tengan una importancia religiosa o histórica.

- Instalaciones comerciales: centros comerciales, edificios de oficinas, estadios deportivos, cualquier otro lugar que pueda albergar a un gran número de personas.

- Fabricación crítica: transformación de materiales en bienes. Esto incluye todos los procesos involucrados en la fabricación y el equipo de transporte. 
- Base de la industria de defensa: instalaciones de producción de recursos militares (por ejemplo, armas, aviones o barcos) y mantenimiento de servicios esenciales (por ejemplo, comunicación) para proteger a una nación.

- Industria nuclear: producción y almacenamiento de sustancias nucleares.

- Espacio: comunicación e investigación.

- Instalaciones de investigación (2015, p. 54).

El lector tal vez se pregunte por la relación de estos elementos con la ciberguerra. El primer aspecto de esta relación ya fue presentado anteriormente y corresponde a la demanda de las TIC en los procesos sociales y cómo se hicieron dependientes estos de las redes tecnológicas. Pero, en segunda medida, esta relación tiene que ver con el hecho de las estructuras precisas que permiten la integración, la modificación y el manejo de los centros de gravedad y principalmente la infraestructura crítica, con el ánimo de mejorar la eficiencia y el control a distancia, la unión de las tecnologías informáticas y el ciberespacio con la humanidad y los actores internacionales.

Esto se ha convertido en una realidad gracias a la invención del sistema de control distribuido (distributed control system [DCs]), como lo es el más conocido de todos, SCADA (supervisory control and data acquisition). Un sistema SCADA se refiere, básicamente, a una red centralizada para la supervisión y adquisición de datos de subestaciones ubicadas en áreas geográficas grandes y distantes. El centro de control es responsable de regular el rendimiento general de todo el sistema y de gestionar su información sensible. Esta gestión depende de la funcionalidad de un conjunto de servidores. Los accesos externos a estos recursos deben ser debidamente abordados y restringidos a través de mecanismos de seguridad, tales como redes inalámbricas, zonas desmilitarizadas (demilitarized zone [DMZ]), sistemas de detección de intrusiones (intrusion detection system [IDS]), sistemas de prevención de intrusiones (intrusion prevention system [IPs]) o antivirus. Algunos de estos accesos pueden provenir de redes corporativas encargadas de reportar evaluaciones estadísticas o 
actualizar planes estratégicos para aumentar la productividad y la producción comercial. Por el contrario, un DCs es un sistema orientado a procesos, cuyo control es limitado desde el punto de vista de la dimensión y distribución geográfica (Alcaraz y Zeadallay, 2015, p. 57).

Hoy en día, las TIC desempeñan un papel crucial en el control y la conectividad entre entidades críticas. Este es el caso de los sistemas SCADA, cuyo control y supervisión dependen, principalmente, de la fiabilidad y seguridad de los canales de comunicación y sistemas de información para enviar, calcular o almacenar comandos, alarmas o mediciones. Se identifican tres principales entidades tecnológicas en esta sección: sistemas de comunicación para grandes distancias, sistemas de comunicación inalámbrica y la influencia de sistema para operaciones de control (Alcaraz y Zeadallay, 2015, p. 59).

En los países desarrollados, y cada vez más en cualquier parte del mundo, las infraestructuras de la información son al mismo tiempo una base fundamental que hace que las sociedades contemporáneas funcionen, así como una fuente de vulnerabilidad. La difusión de las telecomunicaciones, internet y la penetración de las computadoras y las redes informáticas locales aplicadas en tantas facetas de la vida hoy han cambiado todo. Por esta razón, los sistemas de información se consideran un activo crítico, no solo para propósitos de inteligencia tradicionales, sino también para cuestiones de seguridad. Si bien representan una base esencial que permite a las sociedades modernas trabajar y vivir, se han desarrollado sin considerar la seguridad como una prioridad. La recopilación de información ha sido siempre un aspecto crítico e ineludible en la política internacional y las nuevas tecnologías ofrecen herramientas sin precedentes para monitorear los gobiernos, las economías, la defensa y la planificación de la seguridad que pueden convertirse en objetivos de amenazas externas (Colesniuc, 2013, p. 123).

La infraestructura crítica incluye propietarios y operadores públicos y privados, y otras entidades que desempeñan un papel en la protección de la infraestructura de la nación. Los miembros de cada sector de infraestructura crítica realizan funciones que están respaldadas por sistemas de tecnología de la información 
(TI) y control industrial (industrial control system [ICS]). Esta dependencia de la tecnología, la comunicación y la interconectividad de la TI y la ICs ha cambiado y ampliado las vulnerabilidades potenciales y el mayor riesgo potencial para las operaciones. Por ejemplo, a medida que los sistemas de control industrial y los datos producidos en las operaciones de ICS se utilizan cada vez más para prestar servicios críticos y apoyar las decisiones empresariales, deberían considerarse los impactos potenciales de un incidente de seguridad cibernética en los negocios, los activos, la salud y la seguridad de las personas y el medioambiente de una organización. Para gestionar los riesgos de seguridad cibernética, se requiere una comprensión clara de los factores de negocio de la organización y las consideraciones de seguridad específicas para su uso de TI e ICS. Debido a que el riesgo de cada organización es único, junto con su uso de TI e ICs, las herramientas y los métodos utilizados para lograr los resultados variarán ${ }^{14}$. (National Institute of Standards and Technology, 2017, p. 3)

Se considera una serie de factores que aumentan el riesgo de ataque electrónico contra los sistemas informáticos:

- Dificultades inherentes a la seguridad.

- Sistemas insuficientes de concientización y educación de los usuarios y actitudes o prácticas que no cumplen con el manual de procedimientos.

- Disponibilidad de información sobre la penetración de sistemas informáticos sin autorización.

- Reglamentaciones jurídicas poco claras y dificultades jurisdiccionales.

- Intensificación del fenómeno de la globalización (Colesniuc, 2013, p. 124).

14 Traducción del autor. 
La característica más notable de la infraestructura crítica y de las redes informáticas en general es que la seguridad nunca fue una preocupación importante para los primeros desarrolladores e ingenieros de software. Si se echa un vistazo a la historia de internet, todo el mundo es consciente de que su protocolo (el TCP/ $\mathrm{IP}^{15}$ ) nació “abierto”, sin protección o mecanismo de autocifrado. La sencillez y la velocidad eran una prioridad, ya que se suponía que solo una comunidad de científicos muy pequeña la encontraría útil y querría usarla. Sin embargo, cuando llegó el turno para la comunidad de los negocios de acoger y popularizar internet, esas mismas características fueron recibidas con gusto porque significaban más “eficiencia” (desde el punto de vista del tiempo y la velocidad) y menores costos. Las infraestructuras de información crítica son en su mayoría desarrolladas y construidas a nivel nacional, pero también están vinculadas a las infraestructuras de otros países, por lo que el colapso de una parte de la CiI (critical information infrastructure) de un país tendría un serio impacto en la situación de los vecinos ${ }^{16}$. (Colesniuc, 2013, p. 124).

La etapa inicial de toda futura guerra contra un Estado relativamente moderno implicaría cierta forma de guerra cibernética o, más específicamente, ataques cibernéticos a su CII. Aparentemente, la guerra cibernética no sería tan diferente de la guerra electrónica en el pasado. Pero en algunos aspectos la primera es más grande y ha incorporado la segunda. Primero, la guerra electrónica está dirigida solo a bloquear o interrumpir la comunicación del enemigo; la ciberguerra se dirige a sus centros de gravedad, además de obstaculizar las comunicaciones, también puede impedir la circulación y distribución de divisas, energía, gas, agua y el funcionamiento del control del tráfico aéreo y de los servicios de salud. Además, la guerra cibernética tendría efectos tácticos y estratégicos, dependiendo de los recursos empleados, que

15 Transmission control protocol/internet protocol (protocolo de control de transmisión/protocolo de internet).

16 Traducción del autor. 
impedirían la comunicación (y los muchos servicios que dependen de ella) en todo el país (Colesniuc, 2013, p. 125).

La gestión y el monitoreo de los sitios web han mejorado para las instalaciones de la infraestructura crítica, ya que se han conectado progresivamente con internet. La conveniencia adicional de la conectividad, sin embargo, ha convertido la superficie de ataque una vez limitada de estas industrias en un paisaje fértil para los ataques cibernéticos. Debido a los potenciales efectos de alto perfil de los ataques a los sistemas de infraestructura crítica, estas industrias se han convertido en objetivos aún más atractivos para los ciberdelincuentes (Kellermann et al., 2015, p. 6).

Estas infraestructuras han sido protegidas contra ataques físicos y sabotajes durante muchas décadas hasta principios de este siglo. Sin embargo, varios países se dieron cuenta de que muchas de estas infraestructuras críticas también tenían un componente común: dependían en mayor o menor medida de las infraestructuras de información (redes de telecomunicaciones y sistemas informáticos, TIC $)^{17}$. (Kellermann et al., 2015, p. 13)

Es posible llegar a la conclusión de que los centros de gravedad, representados en un concepto más contemporáneo como infraestructuras críticas, se han hecho vulnerables cuando se conectaron al ciberespacio para poder actualizar la forma en la que se desarrollaban los procesos a través de las Tic. Además, una vez conocido que los procesos políticos, económicos, militares y sociales en general se podían llevar a cabo sin determinantes de localización, pero, aparte de eso en tiempo real, fue imposible plantear un mundo sin las TIC.

El ciberespacio, más allá de representar un concepto tecnológico y técnico en las ciencias y disciplinas que le competen, termina siendo un constructo más sociológico en el sentido de que representa una dimensión en la cual pareciera que las personas están y seguirán virtualizando las cosas que antes se desarrollaban exclusivamente en la atmósfera real. En términos equitativos, frente a la idea de las

17 Traducción del autor. 
dimensiones de la guerra, lo que permite entender por qué el ciberespacio se convirtió en otra dimensión para esta práctica es, primero, que este se ha transformado en un ambiente indispensable para dar vida a un mundo globalizado en todos sus aspectos, por lo cual, y ya que el ser humano en su dimensión física sigue amarrado a las distancias geográficas y temporales de la movilidad a lo largo del planeta, la única forma de hacer posible este deseo humano fue en un plano virtual alimentado por la información. Esto claramente llevó a adaptar las cosas que se utilizan para llevar a cabo los procesos de una forma acorde. Segundo, que parte de los procesos a los que se hace referencia involucran actividades que sobrepasan los contextos propios de los actores conectados al ciberespacio, y se configuran en niveles de los Estados nación, por lo que su afectación sería negativa para una sociedad en general, lo que se definió como centro de gravedad e infraestructura crítica.

\section{Las armas en el ciberespacio}

En relación con lo propuesto al inicio del capítulo, solo resta analizar en qué medida se pueden concebir las tecnologías informáticas que permiten la integración al ciberespacio como aquellas capacidades que posibilitan que algunos actores del sistema internacional y actores irregulares puedan atacar la infraestructura crítica en el ciberespacio.

Antes que nada, se debe reconocer que el concepto de arma desde el punto de vista de la ciberguerra solo tiene validez cuando se asume desde la intención del ejecutor de un ataque en el ciberespacio. Asumir el concepto arma desde un principio clásico, es decir, armamento cinético que funcione en las cuatro dimensiones de la guerra previas a esta, no es posible. En la ciberguerra, lo que se emplea son dispositivos capaces de crear, procesar, administrar y enviar información, la cual, en sentido automático, debería interpretarse como una especie de munición en sentido analógico.

Vale la pena recalcar que una herramienta o dispositivo que permita conexión en el ciberespacio, con la suficiente capacidad de manejar programas e información nociva (una computadora), puede ser usada con fines violentos y se transformará en un arma que ataque cualquier 
conexión, por más pequeña que sea, y se infiltre en sistemas, en apariencia seguros (Stein, 2001). En resumen, se necesita la información que se quiera manejar, un terminal y una conexión a la red, es decir, un sistema que ya está ligado en lo más profundo del funcionamiento estatal y global (Criado, Ramilo y Serna, 2002).

Libicki (2009), dentro de la RAND (Research ANd Development), se ha enfocado en la relación entre el ciberespacio y el sector defensa, afirmando el temor — real- que ya existe: un procesador puede ser una amenaza para los Estados que ya usan las tecnológicas informáticas y de comunicación para atacar. Este autor habla de los dispositivos electrónicos desde tres componentes: la capa física, que es por supuesto la que cualquier persona reconoce al ver un aparato electrónico, y todo lo que dentro de él se puede encontrar; este es el instrumento que permite crear una conexión entre el individuo que la maneja y el ciberespacio.

En un segundo momento, se hace referencia a la necesidad del trabajo humano en los procesos tecnológicos, es decir, un nivel donde se ubican las instrucciones que puede un usuario introducir en el dispositivo que tengan un efecto sobre su entorno cibernético. En tercer lugar, se encuentra probablemente el componente más importante, esencial para el funcionamiento del ciberespacio y recurso vital: la información (Libicki, 2009). Si un actor posee habilidades tecnológicas, con la información maliciosa que puede generar, la convierte en términos sintácticos en la medida en que adquiere la capacidad de establecer comportamientos determinados en el sistema que invade.

Un arma cibernética puede afectar gravemente la infraestructura crítica de un Estado, tal y como se constató con la intrusión del programa Stuxnet en el sistema informático del reactor nuclear de Natanz en Irán; ataque que en la actualidad se le atribuye a Israel. Esta es una clase de ataque que ya ha sido revisada en diversas investigaciones y catalogada como una de las formas más poderosas de emplear la informática como un arma (Libicki, 2010).

Muchos de los analistas de la ciberguerra, el ciberespacio y en general las ciberamenazas han apelado a una novela de ciencia ficción para denotar lo que en realidad significa la cibernética, más que la capacidad de administrar información. William Gibson, autor de Neuromancer, 
recreó el concepto de ciberespacio para describir cómo las computadoras y su interconexión estaban generando una red artificial de terminales que dominaban exorbitantes cantidades de información, las cuales podían ser empleadas para diversos fines. Pero lo más importante del relato de Gibson es que las acciones que realizan los humanos se traducen en efectos en el mundo real, y viceversa. Además, y más importante aún, el mundo virtual y físico logran converger de forma tal que las acciones desarrolladas en cada uno de estos tienen repercusiones semejantes en el otro (Gibson, 1984).

En concordancia con la realidad, el general al frente del Comando Estratégico estadounidense, Kevin Chilton, para 2009, dio a conocer al público que los Estados Unidos, al establecer sus operaciones en el contexto transnacional, han entendido que se encuentran actuando en un medioambiente globalizado, caracterizado por la interdependencia, la incertidumbre, la complejidad y los continuos cambios. Como se ha establecido en la Estrategia de Operaciones Militares en el Ciberespacio, la seguridad y el desarrollo de la nación en el mundo interconectado dependen claramente de las Ti e internet como elementos estratégicos para fortalecer y desarrollar los instrumentos del poder nacional (Chilton, 2009, p. 7).

No cabe duda, entonces, de que, al emplear un ciberataque, el fin buscado es que la agresión se transfiera de forma contundente al mundo real, generando efectos psicológicos, caos organizacional y civil, o un ataque dirigido a la infraestructura crítica y, por ende, sobre la población, si se llegara a poner en riesgo el funcionamiento adecuado de ella (Mills, 2010).

Existe una comparación con el mundo de la biología para explicar el comportamiento del ciberespacio. Gutiérrez (2010) explica que los dispositivos que están conectados al ciberespacio pueden ser contrastados con seres vivos, que son invadidos por actores externos en busca de afectar sus estructuras internas (virus), ya sea mediante el ingreso de información errada o el robo de aquella que le permite funcionar.

Esta filtración es denominada ataque, porque busca desequilibrar y acabar con la armonía del sistema, y se puede definir como "una tecnología informática basada en sistemas del mismo orden (software, 
hardware y medio de comunicación) que ha sido diseñada para perjudicar y dañar la estructura y el funcionamiento de algún otro sistema" 18 (Lorents y Ottis, 2010, pp. 131-133). En primera instancia, el ataque de negación de servicio consiste en la generación de un código que se envía en forma imperceptible, a través de herramientas como los virus troyanos o las botnet, a un gran número de computadoras a través de internet (Ghosh, 2004).

Este código, posteriormente, al esconderse en los diversos sistemas interconectados, espera la fecha con la cual fue programado para ejecutar su ataque. Asimismo, transforma la información en una petición de acceso a una página web establecida por el agresor con el fin de que, en el momento de multiplicarse la misma solicitud por el número de computadoras infectadas, el sitio virtual quede deshabilitado para el uso de su administración hasta el punto de imposibilitarlo para seguir prestando sus servicios (Glebocki, 2008).

Otra forma de ataque son las denominadas weapons of mass distraction o armas de distracción masiva, las cuales buscan, a través del empleo de las tecnologías informáticas, manipular a la población civil de forma psicológica. Como lo describe Brenner (2009), las armas de distracción masiva pretenden derrumbar la moral civil con la sofocación de la fe ciudadana en la eficacia de sus gobiernos. Dependiendo del tipo de ataque de manipulación, puede resultar como efecto desde la imposición de un perjuicio o la destrucción de la propiedad (p. 45).

Por otra parte, un ataque de negación del servicio (denial of service [DDos]) tiene una intención muy clara: atacar un destino en el ciberespacio con el fin de interrumpir su servicio y buen funcionamiento, saturarlo de solicitudes para vulnerar su protección y causarle fallas hasta llegar a desactivarlo por completo (Rosenfield, 2009). Eso se logra cuando el sitio ve su capacidad sobrepasada y el encargado de manejarlo se vea imposibilitado para manejarlo y lograr una interacción real con cualquier persona que busca acceder a este espacio (Glebocki, 2008).

18 Traducción del autor. 
A partir de operaciones furtivas, también existen formas de atacar a un Estado a través de la sustracción o robo de información confidencial acerca de su armamento y sistemas de defensa nacionales, planes de guerra, estrategias militares y planeamiento de operaciones (Carr, 2011).

En última instancia, se encuentran los ataques cibernéticos en contra de la infraestructura crítica del Estado, la cual, en caso de verse afectada como resultado de la alteración de sus sistemas informáticos de control con un código malicioso de comando o la generación de un vínculo de control remoto, podría paralizar un Estado y traer consigo efectos devastadores en la vida de sus ciudadanos (Sierra, 2002).

Tan solo es necesario imaginar lo que pasaría si se inhabilitara la red eléctrica de una ciudad y, por ende, no hubiera forma de poner en marcha las bombas de gasolina que proveen de combustible a sistemas de transporte y medios de abastecimiento de elementos esenciales, o que en determinado momento las plantas generadoras de electricidad de los hospitales se agotaran (Ghosh, 2004).

Con lo anterior se entiende el dónde y el con qué, a continuación, se entenderá el cómo, el por qué y, en esencia, el qué. Los siguientes capítulos buscarán exponer cómo se llegó a la ciberguerra y la preparación de los actores para ella, además de sus diversas formas de expresión. 
